\title{
Empowerment bei Kindern und Jugendlichen - die Bedeutung personaler und sozialer Ressourcen und persönlicher Autonomie für die subjektive Gesundheit
}

\section{Empowerment of Children and Adolescents - The Role of Personal and Social Resources and Personal Autonomy for Subjective Health}

Autoren

Institut
M. Erhart, N. Wille, U. Ravens-Sieberer

Klinik und Poliklinik für Kinder- und Jugendpsychosomatik, Zentrum für Geburtshilfe, Kinder- und Jugendmedizin, Universitätsklinikum Hamburg-Eppendorf

\section{Schlüsselwörter \\ - Empowerment \\ - Kinder und Jugendliche \\ - personale, familiäre und soziale Ressourcen \\ - Risiko- und Schutzfaktoren \\ - epidemiologische Studien}

\section{Key words}

- empowerment

- children and adolescents

- personal, familial and social resources

- risks and resilience

- epidemiological studies

\section{Bibliografie}

DOI $10.1055 / s-0028-1103261$ Gesundheitswesen 2008;

70: 721-729

(c) Georg Thieme Verlag KG Stuttgart · New York

ISSN 0941-3790

\section{Korrespondenzadresse}

Prof. Dr. U. Ravens-Sieberer

Universitätsklinikum HamburgEppendorf

Zentrum für Geburtshilfe, Kinder- und Jugendmedizin Klinik für Kinder- und Jugendpsychosomatik Martinistr. 52

20246 Hamburg

Ravens-Sieberer@

uke.uni-hamburg.de

http://child-public-health.de

\section{Zusammenfassung}

$\nabla$

Ziel: Die wissenschaftliche Auseinandersetzung mit dem Empowerment-Konzept fokussiert bisher fast ausschließlich auf die Population der Erwachsenen. Dennoch können Anknüpfungspunkte zur entwicklungspsychologischen Resilienzforschung, zu salutogenetischen sowie Risiken- und Ressourcenkonzepten aufgezeigt werden. In dieser Arbeit soll die Bedeutung personaler, familiärer und sozialer Ressourcen sowie personaler Autonomie als zentrale Zielgrößen von Empowerment für die subjektive Gesundheitseinschätzung untersucht werden.

Methodik: Im Rahmen einer Sekundäranalyse werden die Gesundheitsdaten von 7000 Kindern und Jugendlichen zwischen 10 und 17 Jahren aus der deutschen Health Behaviour in School-aged children (HBSC) Studie sowie 1700 Kindern und Jugendlichen zwischen 11 und 17 Jahren des Studienmoduls Befragung Seelisches Wohlbefinden und Verhalten (BELLA) des Kinder- und Jugendgesundheitssurveys (KiGGS) varianz- und regressionsanalytisch ausgewertet.

Ergebnisse: In den Auswertungen der HBSCStudie erweisen sich ein förderliches Klassenklima sowie schulische Unterstützung durch die Eltern als protektive Faktoren gegenüber selbstberichteten Gesundheitsbeeinträchtigen. In den BELLA/KIGGS-Auswertungen erweisen sich personale, familiäre und soziale Ressourcen sowie persönliche Autonomie als eigenständige Prädiktoren für eine höhere gesundheitsbezogene Lebensqualität (KINDL-R), auch bei Vorliegen psychischer Beeinträchtigungen.

Schlussfolgerung: Die Ergebnisse bestätigen die Wichtigkeit einer Stärkung personaler familiärer und weiterer sozialer Ressourcen sowie die grundsätzliche Bedeutung personaler Autonomie für die Bewältigung von Gesundheitsrisiken und Beeinträchtigungen. Weiterführende explizite Beforschung des Empowerments könnte sich

\section{Abstract \\ V}

Aim: Scientific research on empowerment so far is nearly exclusively focused on the adult population. Nevertheless, it is possible to show a link between empowerment and a) the developmental psychology concepts of resilience, b) autogenetic concepts and c) concepts of risks and resources. This paper aims to study the role of personal, familial and other social resources as well as personal autonomy for subjective healthratings.

Methods: A secondary analysis of the health data of 7000 children and adolescents aged 10-17 years of the German health behaviour in school-aged children (HBSC) study as well as 1700 children aged 11-17 years of the mental health module (BELLA Study) within the German health interview and examination survey for children and adolescents (KiGGS) was performed. Statistical analyses encompassed analyses of variance and linear regression.

Results: Analyses of the HBSC study showed a protective effect for school-class climate as well as parental support, whereby school was associated with fewer self-reported health complaints. Analyses of the BELLA/KiGGS study showed personal, familial and other social resources as well as personal autonomy as unique predictors for a better health-related quality of life (KINDL-R). This was true even if psychological problems were observed.

Conclusion: The results confirm the importance of strengthening personal, familial and other social resources as well as the principal importance of personal autonomy for coping with health risks and health impairments. Future research explicitly focussed on empowerment could relate to the role of personal resources within children's and adolescents' contact with the medical and health care system. It can be expected that strengthening personal resources 
auf die Bedeutung personaler Ressourcen im Kontakt der Kinder und Jugendlichen mit dem Medizin- Helfersystem beziehen. Von einer Stärkung personaler Ressourcen könnte so eine Verbesserung der Kommunikation und ein aktiveres Mitwirkungsvermögen von Kindern und Jugendlichen bei Behandlungsentscheidungen und Bewertungen erwartet werden. benefits and improves the communication and active participation of children and adolescents within treatment-decision and -evaluation.

\section{Einleitung \\ $\nabla$}

\section{Der Ansatz des Empowerments}

Konzentrierte sich die Praxis psychosozialer Arbeit noch vor wenigen Jahrzehnten vornehmlich auf die Defizite der Betroffenen, haben sich in den vergangenen Jahren zunehmend sogenannte Ressourcen-orientierte Ansätze in den Bereichen Sozialpädagogik, psychotherapeutische Arbeit, Gesundheitsförderung, Familienpädagogik und Gemeindepsychologie etabliert. Mit dem Begriff Empowerment werden jene Ansätze bezeichnet, welche darauf zielen, den Betroffenen ihre vorhandenen Fähigkeiten sichtbar zu machen und zu stärken. Angestrebt wird somit die Kräftigung und Freisetzung von Ressourcen und die Stärkung von personalen und sozialen Kompetenzen, mit deren Hilfe selbstbestimmt die eigenen Lebensräume und Lebenswege gestaltet werden können [1]. Empowerment betont dabei, dass die professionelle Dominanz (z.B. von Experten, Ärzten) abgebaut wird und stattdessen die individuelle Entscheidung und Selbstbestimmung gesteigert wird. Empowerment kann als individueller Prozess verstanden werden, durch den die Betroffenen einen Zustand der „Powerlosigkeit“ überwinden und sich zunehmende Kontrolle über ihr Leben sichern [2,3]. Der Empowerment-Prozess startet demnach mit den individuell selbst definierten Bedürfnissen und Bestrebungen und konzentriert sich dann auf die Kapazitätsentwicklung sowie Ressourcen und Unterstützungen, die zur Erreichung dieser Bestrebungen benötigt werden [2]. Als Ziel der Empowerment-Praxis kann damit die Aneignung von Selbstbestimmung und Lebensautonomie durch die Betroffenen angesehen werden.

\section{Empowerment bei Kindern und Jugendlichen}

Diese neue Sichtweise hat in der psychosozialen Arbeit mit Erwachsenen zunehmend an Attraktivität gewonnen und sich etablieren können. Inwieweit diese Etablierung von EmpowermentPrinzipien auch für die Population der Kinder und Jugendlichen gilt, muss jedoch differenzierter beurteilt werden: Zwar hat sich die Auffassung, dass Gesundheit mit der Fähigkeit zur Befriedigung der eigenen Bedürfnisse und der Verwirklichung eigener Bestrebungen zusammenhängt, auch für die Kinder und Jugendlichen durchgesetzt $[4,5]$. Ein zentraler Empowerment Aspekt ist jedoch die Forderung, dass Gesundheitsförderung an jenen Problemen ansetzen muss, welche für die Betroffenen bedeutsam sind - dieser Aspekt wurde für die Population der Kinder lange ignoriert. Kinder wurden als sozial und kognitiv unreif angesehen und dementsprechend zu förderlichem Gesundheitsverhalten angeleitet oder durch entsprechende Gesundheitsdienstleistungen „versorgt“ [4]. Allerdings wird mit der zunehmenden Berücksichtigung von Fragen der subjektiven Gesundheit bzw. gesundheitsbezogenen Lebensqualität von Kindern und Jugendlichen [6,7] die individuelle Perspektive von Kindern und Jugendlichen nun auch bei Behandlungsentscheidungen und -bewertungen anerkannt. Behinderten und benachteiligten Kindern eine „Stimme“ zu geben, stellt eine Schlüsselforderung von zwei Resolutionen der Vereinten Nationen dar [8,9]. Im kli- nischen Setting haben sich Aspekte des Empowerments bei Kindern und Jugendlichen durchgesetzt. Kinder und Jugendliche werden ihrem Entwicklungsstand entsprechend an Versorgungs- und Behandlungsfragen beteiligt, beispielsweise bezüglich der Verordnung von Kontrazeptiva oder der medikamentösen Behandlung von psychiatrischen Erkrankungen.

Eine deutliche Einschränkung betrifft die Verwendung von Empowerment als Forschungsthema bei Kindern und Jugendlichen. Zwar lässt sich bereits für den Anfang der 90er Jahre eine wissenschaftliche Diskussion über die Anwendbarkeit des Empowerment-Konzeptes auf Familien und Kinder erkennen, der Fokus lag dabei jedoch auf einer elterlichen Perspektive [10,11,12]. Nur wenige Arbeiten $[13,4]$ befassten sich mit Empowerment aus einer individuellen Perspektive der Kinder. Auch fast 20 Jahre später hat sich an dieser „Vernachlässigung“ der wissenschaftlichen Nutzung des Empowerment-Konzeptes bei Kindern und Jugendlichen wenig geändert: Eine Literatursuche in der Datenbank der internationalen Psychologischen Literatur ,Psychinfo“ erbringt unter dem Begriff „empowerment“ 1178 Treffer. Die Verknüpfung mit den Suchbegriffen „children“ oder „adolescents“ oder „youth“ weist dagegen nur noch 57 Arbeiten aus. Bei näherer Betrachtung behandeln dabei mehrere Arbeiten [12,14] das Empowerment von Eltern beeinträchtigter Kinder und nicht die Kinder selbst.

Dennoch lassen sich abseits expliziter Empowerment-Forschung Anknüpfungspunkte zu etablierten Forschungskonzepten bei Kindern und Jugendlichen ausmachen: Ein zentraler Aspekt des Empowerments betrifft die Stärkung und Freisetzung von Ressourcen. Hierunter werden nach [15] jene Potenziale verstanden, die von einem Menschen zur Befriedigung seiner Grundbedürfnisse, zum Erreichen langfristiger Identitätszielen sowie zur Bewältigung von Entwicklungsaufgaben und Alltagsanforderungen genutzt werden können. Damit tragen diese Potenziale zur Sicherung der psychischen Integrität und zur Kontrolle von sich selbst und der Umwelt sowie zu einem umfassenden biopsychosozialen Wohlbefinden bei. Nach Willutzki [16] hängt die psychische und physische Gesundheit sowie Wohlbefinden von der Verfügbarkeit und dem Einsatz von Ressourcen ab.

\section{Empowerment und Resilienz bei Kindern und Jugendlichen}

Eine wissenschaftstheoretische Verbindung lässt sich so zur entwicklungspsychologischen Resilienzforschung ausmachen. Diese geht der Frage nach, welche Einflussgrößen betroffene Kinder und Jugendliche bei vorhandenen Belastungen und Risikofaktoren in die Lage versetzen, sich dennoch gesund zu entwickeln [17]. Die so identifizierten Einfluss- oder Schutzfaktoren für die psychische Gesundheit werden grob in personale, familiäre und soziale Ressourcen eingeteilt. Personale Ressourcen sind z. B. der Kohärenzsinn, welcher beinhaltet, a) inwieweit eine Person die von ihr erfahrenen Anforderungen als strukturiert, vorhersagbar und erklärbar wahrnimmt sowie b) die zum Umgang mit diesen Herausforderungen benötigten Ressourcen als verfügbar erlebt und c) diese Herausforderungen als lohnenswert bezüglich des 
eigenen Engagements empfindet $[18,19]$. Der dispositionelle Optimismus bezeichnet die generelle Zuversicht, dass sich Dinge positiv entwickeln, unabhängig von vorausgegangenen Erfahrungen oder eigenen Anstrengungen [20]. Die allgemeine Selbstwirksamkeitserwartung als weitere personale Ressource wird als stabiles Persönlichkeitsmerkmal verstanden und bezeichnet die generelle Überzeugung, selbst über die notwendige Kompetenz zu verfügen, mit Anforderungen umgehen zu können [21]. Bei den familiären Ressourcen spielen insbesondere Aspekte des Familienklimas, d.h. der familiäre Zusammenhalt sowie das Erziehungsverhalten der Eltern eine Rolle [22]. Die sozialen Ressourcen umfassen z.B. die von Gleichaltrigen und Erwachsenen erfahrene oder verfügbare soziale Unterstützung $[23,24]$. Die Stärkung dieser Ressourcen könnte als Zielgröße von Empowerment angesehen werden

Mit dem salutogenetischen Ansatz in den Gesundheitswissenschaften, d.h. der Frage nach der Entstehung von Gesundheit, hat neben der Betrachtung von Risikofaktoren zunehmend die Frage nach Bedingungen und Faktoren, die Gesundheit schützen und zur Widerstandskraft gegenüber Stressoren beitragen, an Bedeutung und Interesse gewonnen [25]. Das epidemiologische Konzept der Risikofaktoren [26] trägt der Tatsache Rechnung, dass in der Regel nicht ein einzelner, genau zu bezeichnender Faktor als eindeutige Erklärung für das Zustandekommen einer chronischen Beeinträchtigung möglich ist, sondern das Zusammenwirken mehrerer Risikofaktoren. Dieses Konzept konnte um das Konzept der sogenannten Schutzfaktoren erweitert werden. Körperliche, psychische und soziale Risikofaktoren schlagen sich dementsprechend nicht „mechanisch“ in entsprechenden beeinträchtigenden Symptomen nieder, sondern erhöhen die Wahrscheinlichkeit entsprechender Beeinträchtigungen. Diese Risikoerhöhung wird jedoch auch maßgeblich durch die einer Person zur Verfügung stehenden Bewältigungsmöglichkeiten und -ressourcen vermittelt ist. Das Konzept der Schutzfaktoren hat zunehmend auch Verbreitung in der Public Health-Forschung bei Kindern und Jugendlichen gefunden $[27,28]$.

Vor dem Hintergrund dieser wissenschaftstheoretischen Anknüpfungspunkte soll in dieser Arbeit aufgezeigt werden, welche Potenziale die Sekundäranalyse bisheriger Studien zur psychosozialen Lage und Gesundheit von Kindern und Jugendlichen unter einer Empowerment-Perspektive bietet. Beispielhaft werden aus zwei großen Gesundheitsstudien ausgewählte Aspekte mit Empowerment-Relevanz analysiert. Diese Arbeit fokussiert dabei auf die Perspektive der Kinder und Jugendlichen und konzentriert sich auf den zentralen Empowerment-Aspekt der Stärkung von Ressourcen. In einer Sekundäranalyse der Health Behaviour in School-aged Children (HBSC) Studie [29] wird die Bedeutung eines förderlichen Klassenklimas sowie schulischer Unterstützung durch die Eltern für die subjektive Gesundheit untersucht. In einer Sekundäranalyse des Studienmoduls Befragung "Seelisches Wohlbefinden und Verhalten“ (BELLA) des Kinder- und Jugendgesundheitssurvey (KiGGS) $[28,30]$ wird die Bedeutung personaler, familiärer und weiterer sozialer Ressourcen sowie das Ausmaß an persönlicher Autonomie für die subjektive Gesundheit und das Wohlbefinden vor dem Hintergrund psychischer Beeinträchtigungen und Behinderungen untersucht. Die Diskussion der so gewonnenen Ergebnisse soll auch die Möglichkeiten und Grenzen der aus Sekundäranalysen erzielbaren Erkenntnisse aufzeigen und den Bedarf nach weiterer expliziter Forschung zum Empowerment-Konzept bei Kindern und Jugendlichen spezifizieren. Der Schwerpunkt dieser Arbeit liegt damit auf einer nicht-medizinisch zentrierten gesundheitswis- senschaftlichen Perspektive. Dieser Ansatz versucht der Bedeutung der Ressourcen im Alltag gerecht zu werten. Die grundsätzliche Veränderbarkeit - beispielsweise personaler Ressourcen zeigt jedoch auch Relevanzen für das Medizinische- und psychosoziale Versorgungssystem auf: Von einer Stärkung personaler Ressourcen könnten so verbesserte Kommunikationsmöglichkeiten und aktivere Partizipationschancen für Kinder und Jugendliche im Kontakt mit der Gesundheitsversorgung erwartet werden.

Diese Arbeit untersucht, ob sich die theoretisch zu erwartende Bedeutung personaler, familiärer und weiterer sozialer Ressourcen in den Indikatoren der subjektiven Gesundheit der HBSC sowie der KiGGS und BELLA Studien nachweisen lässt.

\section{Methodik}

$\nabla$

Studiendesign der HBSC- und KiGGS/BELLA- Studien

Bei der alle vier Jahre durchgeführten HBSC-Studie werden bei 11-, 13- und 15-jährigen Schülern und Schülerinnen Daten über die Gesundheit und das Gesundheitsverhalten erhoben. Die Studie will zu einem erweiterten Verständnis gesundheitsbezogener Einstellungen und Verhaltensweisen junger Menschen beitragen und die Bedingungen ihrer psychosozialen und gesundheitlichen Entwicklung untersuchen [29]. An der aktuellen HBSC-Studie (2005/06) nahmen 41 Länder in Europa, Nordamerika und Israel teil. Deutschland ist mit den Bundesländern Nordrhein-Westfalen, Hessen, Sachsen, Berlin und Hamburg an der HBSC-Studie beteiligt. Die anonyme Datenerhebung erfolgte mittels eines Selbstausfüllfragebogens und fand im Klassenverband statt [31].

Die BELLA-Studie [28] als vertiefendes Studienmodul der KiGGS Studie [30] fokussiert auf die psychische Gesundheit von Kinder und Jugendlichen. Ein weiterer Schwerpunkt der Studie liegt auf der Bedeutung verschiedener individueller, familiärer und sozialer Protektiv- und Risikofaktoren für die mentale Gesundheit. Design und Methode der BELLA Studie sind an anderer Stelle dokumentiert $[28,30,32-34]$. An der BELLA-Studie nahmen Familien mit Kindern zwischen 7 und 17 Jahren teil. In jeder Familie wurde ein Elternteil um die Teilnahme gebeten sowie auch das Kind, sofern es mindestens 11 Jahre alt war. Die Datenerhebung erfolgte mithilfe standardisierter und computerassistierter Telefoninterviews (CATI). Weitere Informationen wurden über einen schriftlichen Fragebogen erhoben, der den Eltern und dem Kind zugeschickt wurde [28].

\section{Stichprobenbeschreibung}

In der deutschen HBSC-Stichprobe liegt die Responserate der Schüler bei $86 \%$, definiert als Anteil der teilnehmenden Schülerinnen und Schüler aus den an der Studie teilnehmenden Schulen. Aus dem so realisierten Datensatz von 11513 Schülerinnen und Schülern wurde ein strukturtypischer Datensatz konstruiert - gewichtet nach der Anzahl der Schüler in öffentlichen Schulen in den fünf Bundesländern (separat für jede der drei Klassenstufen) [31].

Die Teilnahmequote an der KiGGS Studie betrug - bezogen auf alle um Teilnahme gebetene Familien 66,6\%. Von allen 4199 zufällig aus der KiGGS Stichprobe ausgewählten Familien, die um eine Teilnahme an der BELLA-Studie gebeten wurden, erklärten sich $70 \%$ einverstanden. Diejenigen, die ihre Teilnahme zugesagt hatten, nahmen anschließend zu 97\% ( $=2863)$ an der Befragung teil. 


\section{Auswertungsinhalte}

Für die vorliegende Arbeit werden aus der HBSC-Studie folgende Themen mit Empowerment-Relevanz ausgewertet: Die selbsteingeschätzte Gesundheit, ein förderliches Klassenklima und die schulische Unterstützung durch die Eltern. Als weitere Faktoren werden der schulische Erfolg, das selbstberichtete Geschlecht und Alter sowie der familiäre Wohlstand (Family Affluence Scale, FAS) mit berücksichtigt. Weitere Informationen zu diesen Indikatoren können der Veröffentlichung von Currie et al. [29] entnommen werden.

Aus den Informationen mit Empowerment-Relevanz der BELLAStudie werden für diese Arbeit die subjektive Gesundheitseinschätzung nach dem KINDL-R-Lebensqualitätsinstrument ausgewertet [35]. Der KINDL-R erfasst die Dimensionen im „Körperliches Wohlbefinden“, „Emotionales Wohlbefinden“, „Selbstwert“, „Wohlbefinden in der Familie“, „Wohlbefinden in Bezug auf Freunde/Gleichaltrige“ und „Schulisches Wohlbefinden“. Außerdem wird ein übergreifender Messwert der gesundheitsbezogenen Lebensqualität berechnet.

Personale Ressourcen werden über eine aus 5 Items bestehende Skala erfragt [36], deren Items aus der Selbstwirksamkeitsskala von Schwarzer et al. [37], der Optimismusskala des Berner Fragebogens zum Wohlbefinden [38] und der Sense of Coherence Scale [18] stammen.

Soziale Ressourcen werden über die deutsche Übersetzung der „Social Support Scale“ [39] erfasst. Familiäre Schutzfaktoren werden über eine gekürzte Version der Familienklima-Skala [40] zum familiären Zusammenhalt erhoben. Die Skalenwerte dieser drei Ressourcen werden in die Kategorien „unauffällig bzw. normal“, „unterdurchschnittlich bzw. grenzwertig“ und „deutliche Defizite" eingeteilt [36]. Als Indikator der selbst berichteten Autonomie wird die entsprechende Skala des KIDSCREEN-52 Instrumentes [41] ausgewertet.

Als weitere Faktoren werden über den Strengths and Difficulties Questionnaire (SDQ) [42] emotionalen Probleme, Hyperaktivitätsprobleme Probleme mit Gleichaltrigen und Verhaltensprobleme erhobenen. Für den Gesamtproblemwert wurden die Cutoff-Werte der englischen Normstichprobe zugrunde gelegt und die Kinder und Jugendlichen danach als „unauffällig“, „grenzwertig“ oder „auffällig“ klassifiziert.

Der sozioökonomische Status wird über den sogenannten Winkler-Sozialschichtindex ermittelt, welcher die Haushalte in solche mit niedrigem, mittlerem und hohem sozioökonomischen Status klassifiziert [30]. Als soziodemografische Merkmale wurden Alter und Geschlecht erfasst.

\section{Statistische Auswertungen und}

Untersuchungsfragestellungen

Die Reliabilität der aus den HBSC-Studiendaten ad-hoc gebildeten Ressourcenskalen wird über die interne Konsistenz der Itembeantwortung (Cronbachs Alpha) ermittelt. Mittels ANOVA wird analysiert, in welchem Zusammenhang ein förderliches Klassenklima und die Unterstützung der Eltern mit dem schulischen Erfolg, und der allgemeinen Gesundheitseinschätzung stehen. In einer schrittweisen nominalen logistischen Regressionsanalyse wird zunächst analysiert, wie stark schulischer (Miss-)Erfolg mit der Gesundheitseinschätzung assoziiert ist. Im nächsten Schritt werden das Klassenklima und die elterliche Unterstützung als weitere unabhängige Variablen in das Regressionsmodel aufgenommen. Zur Kontrolle sind das Alter, Geschlecht und der sozioökonomische Status als weitere unabhängige Faktoren in beiden Regressionsmodellen enthalten.
Aus der BELLA-Studie wird zunächst mittels ANOVA untersucht, ob Kinder und Jugendliche mit grenzwertigen oder auffälligen emotionalen Problemen oder Verhaltensproblemen im SDQ eine beeinträchtigte Lebensqualität im KINDL berichten. In einer hierarchischen linearen Regressionsanalyse wird für jede Lebensqualitätsskala des KINDL in einem ersten Schritt die Bedeutung des Gesamtwertes emotionaler und Verhaltensprobleme für den entsprechenden Lebensqualitätsaspekt untersucht. In einem weiteren Schritt werden personale, familiäre und sonstige soziale Ressourcen sowie die selbsteingeschätzte Autonomie als weitere Prädiktoren in das Regressionsmodell aufgenommen. Kontrolliert werden diese Analysen für das Alter, Geschlecht und den sozioökonomischen Status.

\section{Ergebnisse \\ $\nabla$}

\section{Auswertungen der HBSC Studie}

Der strukturtypische Datensatz der deutschen HBSC-Studie umfasst 7274 Kinder und Jugendliche zwischen 11 und 15 Jahren. In 0 Tab. 1 werden die wesentlichen Charakteristiken dieser Stichprobe dargestellt.

Die psychometrischen Eigenschaften der ad-hoc Skalenbildungen werden vorab getestet. Die ad-hoc gebildete Skala „Förderliches Klassenklima“ erreicht eine interne Konsistenz von Cronbachs alpha $=0,64$ und liegt damit leicht unterhalb der für Gruppenvergleiche geforderten Grenze von 0,70 [43]. Die interne Konsistenz der ad-hoc gebildeten Skala „Schulische Unterstützung durch Eltern“ beträgt Cronbachs alpha =0,77.

Die in $\bullet$ Tab. 2 dargestellten Ergebnisse der ANOVA zeigen, dass ein förderliches Klassenklima mit den Schulleistungen assoziiert ist: Die durchschnittliche Ausprägung des Klassenklimas erreicht bei „sehr guten“ Schulleistungen 4,3 Punkte, und nimmt bis auf 3,8 Punkten bei unterdurchschnittlichen Schulleistungen ab. Die in $\bullet$ Tab. 2 dargestellten Unterschiede können mit einem „f“" Effektstärkemaß von 0,13 als kleiner Effekt klassifiziert werden [44]. Auch mit der subjektiven Gesundheitseinschätzung zeigt sich eine Assoziation: Bei einer „exzellenten“ Gesundheitseinschätzung liegt die Klassenklimaausprägung im Durchschnitt bei 4,25 Punkten und nimmt bis auf 3,6 Punkte bei „schlechter“ Gesundheitseinschätzung ab. Umgerechnet in das „f" Effektstärkemaß ergibt sich ein Wert von 0,21, der als kleiner Effekt klassifiziert werden kann. Die schulische Unterstützung durch die Eltern liegt bei „sehr guten“ Schulleitungen im Durchschnitt bei

Tab. 1 Strukturtypischer Datensatz für Deutschland nach Bundesland und Klassenstufe, Jungen und Mädchen (HBSC Deutschland 2005/06) [33].

\begin{tabular}{|c|c|c|c|}
\hline & $\begin{array}{l}\text { Gesamt } \\
\text { n }\end{array}$ & $\begin{array}{l}\text { Jungen } \\
\%(n)\end{array}$ & $\begin{array}{l}\text { Mädchen } \\
\text { \% (n) }\end{array}$ \\
\hline \multicolumn{4}{|l|}{ Bundesland } \\
\hline Nordrhein-Westfalen & 4324 & $59,5(2182)$ & $59,4(2142)$ \\
\hline Hessen & 1338 & $19(697)$ & $17,8(641)$ \\
\hline Sachsen & 686 & $8,9(325)$ & $10(361)$ \\
\hline Berlin & 608 & $8,6(315)$ & $8,1(293)$ \\
\hline Hamburg & 318 & $4,1(149)$ & $4,7(169)$ \\
\hline \multicolumn{4}{|l|}{ Klassenstufe } \\
\hline 5. Klasse (Ø Alter $11,4 \pm 0,46$ ) & 2315 & $51(1181)$ & 49 (1134) \\
\hline 7. Klasse (Ø Alter $13,4 \pm 0,50)$ & 2504 & $50,7(1269)$ & $49,3(1235)$ \\
\hline 9. Klasse (Ø Alter $15,4 \pm 0,33$ ) & 2455 & $49,6(1218)$ & $50,4(1237)$ \\
\hline Gesamt & 7274 & $50,4(3668)$ & $49,6(3606)$ \\
\hline
\end{tabular}


Tab. 2 Schulische Erfolg, allgemeine Gesundheitseinschätzung und förderliches Klassenklima sowie schulische Unterstützung durch Eltern.

\begin{tabular}{|c|c|c|c|c|c|c|c|c|c|}
\hline & \multirow[b]{3}{*}{ N } & \multicolumn{4}{|c|}{ Förderliches Klassenklima } & \multicolumn{4}{|c|}{ Schulische Unterstützung durch Eltern } \\
\hline & & & & $95 \%-K I$ & & & & $95 \%-K I$ & \\
\hline & & MW & SD & $\begin{array}{l}\text { Unter- } \\
\text { grenze }\end{array}$ & $\begin{array}{l}\text { Ober- } \\
\text { grenze }\end{array}$ & MW & SD & $\begin{array}{l}\text { Unter- } \\
\text { grenze }\end{array}$ & $\begin{array}{l}\text { Ober- } \\
\text { grenze }\end{array}$ \\
\hline \multicolumn{10}{|l|}{ Schulleistung ${ }^{\mathrm{a}}$} \\
\hline sehr gut & $514-520$ & 4,249 & 0,734 & 4,186 & 4,312 & 4,596 & 0,591 & 4,5449 & 4,647 \\
\hline & $2976-2983$ & 4,176 & 0,655 & 4,153 & 4,200 & 4,575 & 0,549 & 4,5551 & 4,595 \\
\hline \multirow{3}{*}{$\begin{array}{l}\text { durchschnittlich } \\
\text { unterdurchsch. }\end{array}$} & $3273-3281$ & 4,059 & 0,677 & 4,035 & 4,082 & 4,453 & 0,639 & 4,4309 & 4,475 \\
\hline & $261-263$ & 3,800 & 0,822 & 3,699 & 3,900 & 4,186 & 0,858 & 4,0821 & 4,291 \\
\hline & Effektstärke „f“ & 0,133 & & & & 0,144 & & & \\
\hline \multicolumn{10}{|l|}{ Gesundheit $^{\mathrm{b}}$} \\
\hline ausgezeichnet & $2380-2390$ & 4,2545 & 0,634 & 4,2290 & 4,280 & 4,623 & 0,527 & 4,602 & 4,644 \\
\hline gut & $3721-3721$ & 4,0954 & 0,663 & 4,0741 & 4,117 & 4,503 & 0,580 & 4,484 & 4,521 \\
\hline Einigermaßen & $888-888$ & 3,8874 & 0,751 & 3,8379 & 3,937 & 4,281 & 0,769 & 4,231 & 4,332 \\
\hline \multirow[t]{2}{*}{ schlecht } & 99-101 & 3,5825 & 0,989 & 3,3852 & 3,780 & 3,915 & 1,123 & 3,693 & 4,137 \\
\hline & Effektstärke „f“ & 0,196 & & & & 0,210 & & & \\
\hline
\end{tabular}

aWas glaubst du, wie beurteilen deine Lehrerinnen und Lehrer deine Leistungen im Vergleich zu deinen Mitschülern?

bWie würdest du deinen Gesundheitszustand beschreiben?

4,6 Punkten und nimmt bis auf 4,2 Punkte bei „unterdurchschnittlichern“ Schulleistungen ab. Das entsprechende „f“ Effektstärkemaß beträgt 0,14 und entspricht einem kleinen Effekt.

In einem weiteren Auswertungsschritt wurde die Bedeutung der schulischen Leistung für die subjektive Gesundheitseinschätzung analysiert. In einer multinomialen logistischen Regression wurde die Wahrscheinlichkeit einer „guten“, „einigermaßen“ und „schlechten“ anstatt einer „ausgezeichneten“ subjektiven Gesundheitseinschätzung zunächst durch die schulische Leistung erklärt - kontrolliert für Alter und Geschlecht. In einem zweiten Schritt wurden das Klassenklima sowie die schulische Unterstützung durch die Eltern als weitere Prädiktoren in das Regressionsmodell aufgenommen.

- Tab. 3 zeigt, dass schulischer Misserfolg mit einer erhöhten Wahrscheinlichkeit für eine „schlechtere“ subjektive Gesundheit einhergeht. Eine unterdurchschnittliche schulische Leistung erhöht die „Chance“ einer nur „guten“ (Odds ratio [OR]=2,4), „einigermaßen“ (OR=8,9) oder ,schlechten“ (OR=7,9) anstatt einer „sehr guten“ Gesundheitseinschätzung. Eine (nur) „durchschnittliche“ schulische Leistung geht immer noch mit einer erhöhten Chance“ für eine nur „gute“ $(O R=2,2)$ oder „einigermaßen“ (OR =3,7) anstelle einer „sehr guten“ Gesundheitseinschätzung einher. Auch eine nur „gute“ schulische Leistung geht im Vergleich mit einer „sehr guten“ Schulleistung mit einer erhöhten „Chance“ einer nur „guten“ $(O R=1,7)$ oder „einigermaßen“ (OR=1,9) anstelle einer „sehr guten“ Gesundheitseinschätzung einher. Werden das Klassenklima und die schulische Unterstützung durch die Eltern als weitere Prädiktoren in das Regressionsmodell aufgenommen, dann verkleinern sich diese Koeffizienten, d.h. ein Teil des Erklärungsbeitrages schulischen Misserfolgs für die Gesundheit wird über ein ungünstiges Klassenklima und schwache elterliche Unterstützung erklärt. Gleichzeitig erweisen sich ein förderliches Klassenklima und die schulische Unterstützung durch die Eltern auch im Zusammenhang mit den anderen Prädiktoren (Alter, Geschlecht, sozioökonomischer Status, schulischer Erfolg) als eigenständig prädiktive Faktoren für ein geringeres Risiko einer nur „guten“ ( $O R=0,7$ bzw. 0,8), „einigermaßen“ (OR=0,5 bzw. 0,5) oder „schlechten“
(OR=0,4 bzw. 0,4) anstatt einer „sehr guten“ Gesundheitseinschätzung.

\section{Auswertungen der BELLA-Studie}

Die vorliegenden Auswertungen der BELLA-Studie beziehen sich auf die 1722 Kinder und Jugendlichen im Alter von 11-17 Jahren. $\odot$ Tab. 4 zeigt die Zusammensetzung dieser Stichprobe nach soziodemografischen Merkmalen.

Kinder und Jugendliche mit grenzwertigem und insbesondere auffälligem Befund im SDQ-Gesamtproblemwert berichten eine verminderte subjektive Gesundheit bzw. Lebensqualität im KINDL. Der stärkste Effekt findet sich im Gesamtwert des KINDL und kann mit einem „f“ Effektstärkemaß von 0,44 als starker Effekt klassifiziert werden [44].

Die Bedeutung emotionaler Probleme und Verhaltensprobleme für die subjektive Gesundheitseinschätzungen wurde zusätzlich in linearen Regressionsanalysen der KINDL-Skalen auf den nicht kategorisierten SDQ-Gesamtproblemwert, sowie das Alter, Geschlecht und den sozioökonomischen Status untersucht. $\bullet$ Tab. 5 zeigt die Ergebnisse: Für alle KINDL-Skalen resultieren statistisch signifikante Regressionskoeffizienten, die eine geringere Lebensqualität bei stärkerer psychischer Problembelastung indizieren. Werden die familiären, personalen und sozialen Ressourcen sowie persönliche Autonomie als weitere Prädiktoren in die Regressionsgleichungen aufgenommen, resultieren geringere Regressionskoeffizienten für den SDQ-Gesamtwert, d.h. ein Teil der Bedeutung emotionaler und Verhaltensprobleme für Beeinträchtigungen der Lebensqualität kann somit über die mit solchen Auffälligkeiten assoziierten Defizite in den familiären, personalen und sozialen Ressourcen sowie mangelnde Autonomie erklärt werden. Die familiären Ressourcen sind für alle Lebensqualitätsbereiche außer der KINDL-Skala „Freunde“ ein statistisch signifikanter Prädiktor. Die Regressionskoeffizienten indizieren bessere Lebensqualitätseinschätzungen bei stärker ausgeprägten Ressourcen. Die personalen Ressourcen stellen für alle KINDL-Skalen außer den Skalen „Familie“ und „Körper“ eine statistisch signifikante Erklärungsgröße dar. Die Regressionskoeffizienten indizieren ebenfalls eine mit höherer Ressourcenausstattung assoziierte „bessere“ Lebensqualität. Im Zusammenhang mit allen anderen Prädiktoren leisten auch die sozialen 
Tab. 3 Schulische Leistungen, allgemeine Gesundheitseinschätzung und förderliches Klassenklima.

\begin{tabular}{|c|c|c|c|c|c|c|c|}
\hline \multirow{2}{*}{$\begin{array}{l}\text { Kriterium } \\
\text { Gesundheits- } \\
\text { einschätzunga }\end{array}$} & \multirow[t]{2}{*}{ Prädiktoren } & \multicolumn{3}{|c|}{ Model 1} & \multicolumn{3}{|c|}{ Model 2} \\
\hline & & OR & $\begin{array}{l}95 \% \text { KI } \\
\text { Unter }\end{array}$ & $\begin{array}{l}95 \% \mathrm{KI} \\
\text { Ober }\end{array}$ & OR & $\begin{array}{l}95 \% \text { KI } \\
\text { Unter }\end{array}$ & $\begin{array}{l}95 \% \mathrm{KI} \\
\text { Ober }\end{array}$ \\
\hline \multirow[t]{5}{*}{ gut } & $\begin{array}{l}\text { männlich } \\
\text { weiblich }^{\text {b }}\end{array}$ & $\begin{array}{l}0,534 \\
.\end{array}$ & 0,480 & 0,595 & 0,494 & $\begin{array}{l}0,442 \\
\text {. }\end{array}$ & 0,552 \\
\hline & $\begin{array}{l}\text { 11-Jahre } \\
\text { 13-Jahre } \\
\text { 15-Jahre }\end{array}$ & $\begin{array}{l}0,793 \\
0,963 \\
.\end{array}$ & $\begin{array}{l}0,694 \\
0,845 \\
.\end{array}$ & $\begin{array}{l}0,906 \\
1,096 \\
.\end{array}$ & $\begin{array}{l}0,914 \\
1,043 \\
\cdot\end{array}$ & $\begin{array}{l}0,795 \\
0,913 \\
.\end{array}$ & $\begin{array}{l}1,051 \\
1,192 \\
.\end{array}$ \\
\hline & $\begin{array}{l}F^{F S^{\mathrm{C}}}=\text { niedrig } \\
\mathrm{FAS}^{\mathrm{c}}=\text { mittel } \\
\mathrm{FAS}^{\mathrm{c}}=\text { hoch }^{\mathrm{b}}\end{array}$ & $\begin{array}{l}1,489 \\
1,239 \\
.\end{array}$ & $\begin{array}{l}1,279 \\
1,083 \\
.\end{array}$ & $\begin{array}{l}1,732 \\
1,418 \\
.\end{array}$ & $\begin{array}{l}1,448 \\
1,232 \\
.\end{array}$ & $\begin{array}{l}1,239 \\
1,074 \\
.\end{array}$ & $\begin{array}{l}1,691 \\
1,414 \\
.\end{array}$ \\
\hline & $\begin{array}{l}\text { schulische Leistung } \\
\text { - unterdurchschnittlich } \\
\text { - durchschnittlich } \\
\text { - gut } \\
\text { - sehr gut }\end{array}$ & $\begin{array}{l}2,425 \\
2,213 \\
1,701 \\
.\end{array}$ & $\begin{array}{l}1,664 \\
1,805 \\
1,390 \\
.\end{array}$ & $\begin{array}{l}3,535 \\
2,714 \\
2,081 \\
.\end{array}$ & $\begin{array}{l}2,093 \\
2,077 \\
1,642 \\
\end{array}$ & $\begin{array}{l}1,424 \\
1,685 \\
1,336\end{array}$ & $\begin{array}{c}3,075 \\
2,559 \\
2,019 \\
.\end{array}$ \\
\hline & $\begin{array}{l}\text { Klassenklima } \\
\text { Unterstützung Eltern }\end{array}$ & & & & $\begin{array}{l}0,698 \\
0,753\end{array}$ & $\begin{array}{l}0,638 \\
0,677\end{array}$ & $\begin{array}{l}0,763 \\
0,838\end{array}$ \\
\hline \multirow[t]{5}{*}{ einigermaßen } & $\begin{array}{l}\text { männlich } \\
\text { weiblich }{ }^{\mathrm{b}}\end{array}$ & $\begin{array}{l}0,448 \\
.\end{array}$ & 0,381 & 0,528 & 0,394 & 0,332 & 0,467 \\
\hline & $\begin{array}{l}\text { 11-Jahre } \\
\text { 13-Jahre } \\
\text { 15-Jahre }\end{array}$ & $\begin{array}{l}0,649 \\
0,894 \\
.\end{array}$ & $\begin{array}{l}0,528 \\
0,741 \\
.\end{array}$ & $\begin{array}{l}0,799 \\
1,078 \\
.\end{array}$ & $\begin{array}{l}0,875 \\
1,057 \\
.\end{array}$ & $\begin{array}{l}0,703 \\
0,868 \\
.\end{array}$ & $\begin{array}{l}1,090 \\
1,286 \\
.\end{array}$ \\
\hline & $\begin{array}{l}F A S^{\mathrm{C}}=\text { niedrig } \\
F A S^{\mathrm{C}}=\text { mittel } \\
F A S^{\mathrm{C}}=\text { hoch }^{\mathrm{b}}\end{array}$ & $\begin{array}{l}1,935 \\
1,216 \\
.\end{array}$ & $\begin{array}{l}1,537 \\
0,978 \\
.\end{array}$ & $\begin{array}{l}2,437 \\
1,511 \\
.\end{array}$ & $\begin{array}{l}1,681 \\
1,171 \\
.\end{array}$ & $\begin{array}{l}1,323 \\
0,936 \\
.\end{array}$ & $\begin{array}{l}2,138 \\
1,465 \\
.\end{array}$ \\
\hline & $\begin{array}{l}\text { schulische Leistung } \\
\text { - unterdurchschnittlich } \\
\text { - durchschnittlich } \\
\text { - gut } \\
\text { - sehr gut }\end{array}$ & $\begin{array}{l}8,915 \\
3,677 \\
1,937\end{array}$ & $\begin{array}{l}5,337 \\
2,514 \\
1,318\end{array}$ & $\begin{array}{l}14,893 \\
5,377 \\
2,846\end{array}$ & $\begin{array}{l}6,317 \\
3,187 \\
1,827 \\
.\end{array}$ & $\begin{array}{l}3,708 \\
2,155 \\
1,229 \\
.\end{array}$ & $\begin{array}{r}10,764 \\
4,714 \\
2,716 \\
.\end{array}$ \\
\hline & $\begin{array}{l}\text { Klassenklima } \\
\text { Unterstützung Eltern }\end{array}$ & & & & $\begin{array}{l}0,507 \\
0,532\end{array}$ & $\begin{array}{l}0,449 \\
0,465\end{array}$ & $\begin{array}{l}0,573 \\
0,609\end{array}$ \\
\hline \multirow[t]{6}{*}{ schlecht } & $\begin{array}{l}\text { männlich } \\
\text { weiblich }{ }^{\mathrm{b}}\end{array}$ & 0,524 & 0,345 & 0,797 & 0,461 & 0,299 & 0,709 \\
\hline & $\begin{array}{l}\text { 11-Jahre } \\
\text { 13-Jahre } \\
\text { 15-Jahreb }\end{array}$ & $\begin{array}{l}0,580 \\
0,800 \\
.\end{array}$ & $\begin{array}{l}0,334 \\
0,497 \\
.\end{array}$ & $\begin{array}{l}1,005 \\
1,286 \\
.\end{array}$ & $\begin{array}{l}1,020 \\
0,995 \\
.\end{array}$ & $\begin{array}{l}0,580 \\
0,606 \\
.\end{array}$ & $\begin{array}{l}1,796 \\
1,634 \\
\cdot\end{array}$ \\
\hline & $\begin{array}{l}\text { FAS }^{\mathrm{c}}=\text { niedrig } \\
\text { FAS }^{\mathrm{c}}=\text { mittel } \\
\mathrm{FAS}^{\mathrm{c}}=\text { hoch }^{\mathrm{b}}\end{array}$ & $\begin{array}{l}2,158 \\
1,178\end{array}$ & $\begin{array}{l}1,186 \\
0,651\end{array}$ & $\begin{array}{c}3,924 \\
2,134 \\
.\end{array}$ & $\begin{array}{l}1,562 \\
1,074 \\
\cdot\end{array}$ & $\begin{array}{l}0,841 \\
0,588 \\
.\end{array}$ & $\begin{array}{l}2,903 \\
1,963 \\
.\end{array}$ \\
\hline & $\begin{array}{l}\text { schulische Leistung } \\
\text { - unterdurchschnittlich } \\
\text { - durchschnittlich } \\
\text { - gut } \\
\text { - sehr gut }\end{array}$ & $\begin{array}{l}7,870 \\
1,201 \\
0,713\end{array}$ & $\begin{array}{l}3,277 \\
0,572 \\
0,330\end{array}$ & $\begin{array}{l}18,904 \\
2,521 \\
1,537\end{array}$ & $\begin{array}{l}6,246 \\
1,298 \\
0,861 \\
.\end{array}$ & $\begin{array}{l}2,381 \\
0,566 \\
0,366 \\
.\end{array}$ & $\begin{array}{r}16,387 \\
2,977 \\
2,029 \\
.\end{array}$ \\
\hline & $\begin{array}{l}\text { Klassenklima } \\
\text { Unterstützung Eltern }\end{array}$ & & & & $\begin{array}{l}0,365 \\
0,366\end{array}$ & $\begin{array}{l}0,284 \\
0,286\end{array}$ & $\begin{array}{l}0,470 \\
0,469\end{array}$ \\
\hline & Nagelkerke Pseudo R² & 0,076 & & & 0,127 & & \\
\hline
\end{tabular}

${ }^{a}$ Wie würdest du deinen Gesundheitszustand beschreiben? (Referenzkategorie: ausgezeichnet).

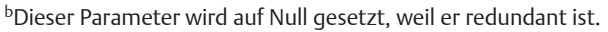

'FAS: Family Affluence Scale [32].

Ressourcen für alle Lebensqualitätsbereiche außer KINDL-Skala „Körper“ und „Schule“ einen statistisch signifikanten Erklärungsbeitrag: Stärker ausgeprägte Ressourcen sind mit höheren Lebensqualitätseinschätzungen assoziiert. Auch die persönliche Autonomie leistet im Zusammenhang mit den anderen Faktoren einen eigenständigen, statistisch signifikanten Erklärungsbeitrag zu allen KINDL Skalen außer Selbstwert und Schule: Je stärker ausgeprägt die Autonomie ist, desto besser wird die Lebensqualität beurteilt. Durch die Hinzunahme der drei Ressourcenskalen und der Autonomie erhöht sich der insgesamt erklärte Varianzanteil zwischen +0,03 (KINDL „Körper“) bis +0,16 (KINDL Total und „Familie“) ( $\bullet$ Tab. 5).
Tab. 4 Stichprobenbeschreibung des ungewichteten Datensatzes der BELLA-Studie, nur 11- bis 17-Jährige.

\begin{tabular}{|c|c|c|c|}
\hline & $\begin{array}{l}\text { Gesamt } \\
\text { n }\end{array}$ & $\begin{array}{l}\text { Jungen } \\
\%(n)\end{array}$ & $\begin{array}{l}\text { Mädchen } \\
\text { \% (n) }\end{array}$ \\
\hline \multicolumn{4}{|l|}{ Region } \\
\hline Ehemalige BRD & 1078 & $63,6(547)$ & $61,7(531)$ \\
\hline Ehemalige DDR & 643 & $36,4(313)$ & $38,3(330)$ \\
\hline \multicolumn{4}{|l|}{ Altersgruppen } \\
\hline $11-13$ Jahre ( $\varnothing$ Alter $12,0 \pm 0,85$ ) & 780 & $46,4(362)$ & $53,6(418)$ \\
\hline 14-17 Jahre ( $\varnothing$ Alter $15,5 \pm 1,10$ ) & 941 & $53,0(499)$ & $47,0(442)$ \\
\hline Gesamt & 1721 & $50,0(861)$ & $50,0(860)$ \\
\hline
\end{tabular}


Tab. 5 Lineare multiple Regression der Lebensqualitätseinschätzungen auf psychische Probleme und familiäre, personale und soziale Ressourcen: Standardisierte Regressionskoeffizienten (beta) und erklärter Varianzanteil $\left(R^{2}\right)$

\begin{tabular}{|c|c|c|c|c|c|c|c|}
\hline & $\begin{array}{l}\text { KINDL } \\
\text { Total } \\
\text { beta }\end{array}$ & $\begin{array}{l}\text { KINDL } \\
\text { Körper } \\
\text { beta }\end{array}$ & $\begin{array}{l}\text { KINDL } \\
\text { Psyche } \\
\text { beta }\end{array}$ & $\begin{array}{l}\text { KINDL } \\
\text { Selbst-wert } \\
\text { beta }\end{array}$ & $\begin{array}{l}\text { KINDL } \\
\text { Familie } \\
\text { beta }\end{array}$ & $\begin{array}{l}\text { KINDL } \\
\text { Freunde } \\
\text { beta }\end{array}$ & $\begin{array}{l}\text { KINDL } \\
\text { Schule } \\
\text { beta }\end{array}$ \\
\hline \multicolumn{8}{|l|}{ Modell 1} \\
\hline Alter &,$- 142^{* *}$ &,$- 152^{* *}$ &,$- 110^{* *}$ &, $122^{* *}$ &,$- 084^{* *}$ &,$- 152^{* *}$ &,$- 207^{* *}$ \\
\hline geschlecht &,$- 054^{* *}$ &,$- 147^{* *}$ &,- 008 &,$- 082^{* *}$ &,- 016 &,- 020 &, $060 * *$ \\
\hline soziale Schicht $\mathrm{n}$. Winkler & ,006 &,- 015 &,- 023 &, $000^{*}$ & ,004 &,$- 057^{*}$ &, $104^{* *}$ \\
\hline Gesamtproblemwert SDQ &,$- 603^{* *}$ &,$- 415^{* *}$ &,$- 429 * *$ &,$- 279^{* *}$ &,$- 370^{* *}$ &,$- 387^{* *}$ &,$- 476^{* *}$ \\
\hline adjustiertes $\mathrm{R}^{2}$ & 0,398 & 0,232 & 0,196 & 0,097 & 0,145 & 0,174 & 0,294 \\
\hline \multicolumn{8}{|l|}{ Modell 2} \\
\hline alter &,$- 125^{* *}$ &,$- 129^{* *}$ &,$- 117^{* *}$ &, $124^{* *}$ &,- 026 &,$- 170^{* *}$ &,$- 203^{* *}$ \\
\hline Geschlecht &,$- 092^{* *}$ &,$- 146^{* *}$ &,$- 057^{*}$ &,$- 110^{* *}$ &,- 030 &,$- 080^{* *}$ &, $056^{*}$ \\
\hline soziale Schicht n. Winkler &,- 008 &,- 010 &,- 037 &,- 019 &,- 003 &,$- 067^{* *}$ &, $095^{* *}$ \\
\hline Gesamtproblemwert SDQ &,$- 392^{* *}$ &,$- 329^{* *}$ &,$- 265^{* *}$ &,$- 111^{* *}$ &,$- 214^{* *}$ &,$- 240^{* *}$ &,$- 374^{* *}$ \\
\hline familiäre Ressourcen ${ }^{\mathrm{a}}$ &, $198^{* *}$ &, $104^{* *}$ &, $076^{* *}$ &, $112^{* *}$ &, $386^{* *}$ &, 032 &, $051^{*}$ \\
\hline personale Ressourcen ${ }^{b}$ &, $168^{* *}$ &, 045 &, $148^{* *}$ &, $231^{* *}$ &,- 051 &, $096 * *$ &, $182^{* *}$ \\
\hline soziale Ressourcenc &, $157^{* *}$ &, 000 &, $209^{* *}$ &, $100^{* *}$ &, $074^{* *}$ &, $264^{* *}$ &, 003 \\
\hline Autonomie &, $126^{* *}$ &, $115^{* *}$ &, $092^{* *}$ &, 045 &, $091^{* *}$ &, $109^{* *}$ &, 043 \\
\hline adjustiertes $\mathrm{R}^{2}$ & 0,560 & 0,260 & 0,307 & 0,198 & 0,302 & 0,287 & 0,332 \\
\hline
\end{tabular}

${ }^{*} \mathrm{p}<0,05 ;{ }^{* *} \mathrm{p}<0,01$

aFamilienklimaskala [42]

'Kernitems Personale Ressourcen der KiGGS/BELLA Studie [38]

'Social Support Scale [41]

\section{Diskussion}

Im Ergebnis lässt sich die Bedeutung der personaler, familiärer, schulischer und sozialer Ressourcen sowie persönlicher Autonomie für die subjektive Gesundheit in zwei verschiedenen Kontexten nachweisen: Ein förderliches Klassenklima und schulische Unterstützungen durch die Eltern sind nicht nur mit der schulischen Leistung, sondern auch mit der selbsteingeschätzten Gesundheit assoziiert. Die vertiefenden multivariaten Auswertungen zeigen, dass ein förderliches Klassenklima sowie eine gut ausgeprägte schulische Unterstützung durch die Eltern, unabhängig vom schulischen Erfolg oder Misserfolg, positiv mit einer besseren subjektiven Gesundheit assoziiert sind. Auch wenn die kausale Wirkrichtung anhand der hier analysierten Querschnittsdaten nicht geklärt werden kann - und die aufgedeckten Zusammenhänge somit eventuell Ausdruck allgemeiner Zufriedenheits- bzw. Somatisierungstendenzen sind - könnten die Ergebnisse auch so interpretiert werden, dass eine Stärkung eines förderlichen Klassenklimas und eine Verbesserung der elterlichen Unterstützung sich gesundheitlich protektiv auswirken und den (in den multivariaten Analysen aufgezeigten) gesundheitlichen Risiken schulischen Misserfolges entgegen wirken können. In bisherigen Studien zur Bedeutung schulischer Aspekte für die Schülergesundheit erwies sich ein fehlendes Zugehörigkeitsgefühl [45], fehlende gegenseitige Unterstützung unter den Schülern [46] sowie konflikthafte Beziehungen/ Schikanieren [47] als bedeutende Faktoren für das Auftreten psychischer Erkrankungen und Störungen. Die vorliegenden Befunde bestätigen auch die Ergebnisse anderer Studien über die Bedeutung familiärer Ressourcen, insbesondere für die psychische Gesundheit von Kindern und Jugendlichen [28]. Masten und Reed [48] nennen als protektive Faktoren innerhalb der Familie unter anderem eine enge Beziehung des Kindes zu fürsorglichen Eltern sowie ein positives Familienklima mit geringen Konflikten. Möglicherweise ist eine gut ausgeprägte schulische Unterstützung durch die Eltern nicht nur per se ein protektiver
Faktor, sondern kann als Indikator für ein dahinterstehendes positives Familienklima und eine gute Beziehung zu den Eltern fungieren. Inwieweit schulische Unterstützung durch die Eltern die negativen Auswirkungen schulischer Belastungen abzupuffern vermag und zu einer produktiveren Verarbeitung dieser Anforderungen führen kann [49] muss in weiteren Datenanalysen statistisch überprüft werden.

Auch die Auswertungen der BELLA-Studie bestätigen die Bedeutung personaler, familiärer und sozialer Ressourcen als wichtige Zielgrößen des Empowerments für die Lebensqualität von Kindern und Jugendlichen. Je stärker ausgeprägt diese Aspekte sind, desto besser wird die eigene Lebensqualität beurteilt. In bisherigen Studien konnte die protektive Wirkung der Ressourcenfaktoren für das Auftreten psychischer Störungen und Probleme bereits nachgewiesen werden $[48,28]$. Darüber hinausgehend weisen unsere Ergebnisse darauf hin, dass die Stärkung dieser Aspekte auch den negativen Auswirkungen von psychischen Behinderungen entgegen wirken kann, da zumindest ein Teil deren Beeinträchtigenden Wirkung über eine Konfundierung mit einer schlechten Ressourcenausstattung erklärt werden kann. Vermutet werden kann außerdem auch eine Stärkung der eigenen Kompetenz im Umgang mit diesen Problemen [49]. Eine gut ausgeprägte Autonomie kann nach unseren Ergebnissen ebenfalls als protektiver Faktor für die subjektive Gesundheit und Wohlbefinden angesehen werden und weist auf die Bedeutung der „Selbstbemächtigung“ der Betroffenen für den Umgang und die Bewältigung auch psychischer Probleme und Behinderungen.

Limitierungen dieser Arbeit betreffen die Analyse von Querschnittsdaten, die einerseits keine Rückschlüsse auf kausale Wirkungen zulassen, andererseits den grundsätzlichen prozesshaften Charakter von Empowerment nur schwer berücksichtigen können [3]. Eine weitere grundsätzliche Einschränkung betrifft die hier durchgeführte Sekundäranalyse vorhandener Daten aus epidemiologischen Studien über Kinder und Jugendliche. In Ermangelung klinischer Falldefinitionen musste etwa da- 
rauf verzichtet werden, die Bedeutung der Empowerment-Aspekte bei definierten klinischen Fällen zu untersuchen. Stattdessen wurde die im Wesentlichen mittels Screening-Instrumenten erhobenen Gesundheitsinformationen in die Analysen einbezogen. Auch die Identifikation von definierten Fällen, die als „powerless“ spezifischer Aufmerksamkeit bedürften, ist aus den vorhandenen Informationen nur eingeschränkt zu leisten, da insbesondere Informationen zu Partizipations- und Kontrollmöglichkeiten etwa der eigenen Gesundheitsversorgung fehlen - zwei Aspekte mit zentraler Relevanz für Empowerment [4]. Konkret erfahrenes Empowerment, z.B. in Form von Beratung und Unterstützungsmanagement [50] oder der Weiterentwicklung von förderlichen Netzwerkstrukturen [51] konnte nicht untersucht werden. Grundsätzlich erscheint es so nicht möglich, auch nur wenigstens die wesentlichen Hauptaspekte des Empowement-Konzeptes zu berücksichtigen. So kann beispielsweise die Frage nach der Bedeutung von Empowerment im Kontakt mit dem Medizin-/Helfersystem im Rahmen der ausgewerteten Studien nicht untersucht werden. Auch die Operationalisierung der ausgewerteten Empowerment Aspekte weist Grenzen auf: Beispielsweise fokussiert die hier ausgewertete Skala Autonomie auf Aspekte der Freizeitgestaltung und des Zeitmanagement und erfasst damit nur Teilbereiche persönlicher Lebensautonomie. Kritisch zu betrachten ist auch die Operationalisierung personaler Ressourcen als globaler Index, wodurch die Ableitung von spezifischen Maßnahmen zur Stärkung dieser Zielgrößen des Empowerments erschwert wird.

Als Fazit ist somit eine weiterführende - explizit auf die Empowerment-Thematik ausgerichtete - Forschung bei Kindern und Jugendlichen mit spezifischen Erhebungsinstrumenten zu fordern. Insbesondere die Berücksichtigung des prozesshaften $\mathrm{Ge}-$ schehens von EmpowermenterfordertentsprechendeStudiendesigns, wie z. B. Längsschnittstudien. Im Sinne einer Mehrebenenperspektive sollte dabei sowohl die Individualebene der betroffenen Kinder und Jugendlichen als auch die näheren und erweiterten Kontexte berücksichtigt werden, da individuelle Veränderungen kaum unabhängig von Veränderungen in näheren und erweiterten sozialen Kontexten wie z.B. Familie, Peergruppe, Schule, Gemeinde sowie weitere gesellschaftliche und politische Rahmenbedingungen untersucht werden können [52]. Unabhängig von den genannten methodischen Limitierungen weisen die gefundenen Ergebnisse jedoch auf das Potential von Empowerment bei Kindern und Jugendlichen hin. Insbesondere die Stärkung von personalen, familiären und sozialen Ressourcen sowie der Aufbau persönlicher Autonomie stellen geeignete potenzielle Zielgrößen dar, die im Rahmen von Präventionsmaßnahmen gestärkt werden sollten.

\section{Literatur}

1 Herriger N. Empowerment in der Sozialen Arbeit. Eine Einführung. 2. Auflage. Stuttgart: Kohlhammer; 2002

2 Arai S. Empowerment: From the theoretical to the personal. Journla of Leisurability 1997; 24: 1

3 Perkins DD, Zimmerman MA. Empowerment theory, research, and application. American Journal of Community Psychology 1995; 23 (5): 569-579

4 Kalnins I, McQueen DV, Backet KC et al. Children, empowerment and health promotion: some new directions in research and practice. Health Promotion International 1992; 7: 53-59

5 Edwards TC, Huebner CE, Conell FA et al. Adolescents quality of life, Part I: conceptual and measurement model. Journal of Adolescence 2002; 25: 275-286

6 Varni JW, Burwinkle TM, Lane MM. Health-related quality of life measurement in pediatric clinical practice: An appraisal and precept for future research and application. Health and Quality of Life Outcomes 2005; 3 (1): 34

7 Ravens-Sieberer $U$, Erhart $M$, Wille $N$ et al. Generic health-related quality of life assessment in children and adolescents: methodological considerations. Pharmacoeconomics 2006; 24 (12): 1199-1220

8 United Nations. Convention on the rights of the child. New York: United Nations; 1989

9 United Nations. Convention on the rights of persons with disabilities. Resolution 60/232. New York: United Nations; 2006

10 Dunst $C J$, Paget KD. Parent-professional partnerships and family empowerment. In: Fine MJ, Hrsg. Collaboration with parents of exceptional children. Brandon, VT: Clinical Psychology Publishing; 1991; 25-44

11 Dunst CJ, Trivette CM. Enabling and empowering families: Conceptual and intervention issues. School Psychology Review 1987; 16: 443456

12 Hegar RL. Empowerment-based practice with children. Social Services Review 1989; 63: 372-383

13 Koren PE, DeChillo N, Friesen BJ. Measuring empowerment in Families whose children have emotional disabilities:A brief questionnaire. Rehabilitation Psychology 1992; 37 (4): 305-321

14 Nachshen JS, Minnes P. Empowerment in parents of school-aged children with and without developmental disabilities. Journal of Intellectual Disability Research 2005; 49 (12): 889-904

15 Herriger $N$. Ressourcendiagnostik und Ressourcenaktivierung in der Sozialen Arbeit, unveröffentl. Manuskript. Düsseldorf; 2005

16 Willutzki $U$. Ressourcen. Einige Bemerkungen zur Begriffsklärung. In: Schemmel H, Schaller J, Hrsg. Ressourcen. Ein Hand- und Lesebuch zur therapeutischen Arbeit. Tübingen: DGVT-Verlag; 2003; 91-109

17 Masten AS, Hubbard JJ, Gest SD et al. Competence in the context of adversity: Pathways to resilience and maladaptation from childhood to late adolescence. Dev Psychopathol 1999; 11: 143-169

18 Antonovsky A. The structure and properties of the Sense of Coherence Scale. Social Science and Medicine 1993; 36: 725-733

19 Bengel J, Strittmatter R, Willmann H. Was erhält Menschen gesund? Antonovskys Modell der Salutogenese - Diskussionsstand und Stellenwert. Köln: Bundeszentrale für gesundheitliche Aufklärung; 1998; 6

20 Scheier MF, Carver CS. Optimism, coping and health: Assessment and implications of generalized outcome expectancies. Health Psychology 1985; 4: 219-247

21 Schwarzer R. Optimistische Kompetenzerwartung: Zur Erfassung einer personalen Bewältigungsressource. Diagnostica 1994; 40: 105-123

22 Darling N. Parenting style and its correlates. ERIC Digest EDO-PS-99-3, Clearinghouse on Elementary and Early Childhood Education. Illinois: University of Illinois; 1999

23 Ezzel CE, Cupit Swenson C, Brondino MJ. The relationship of social support to physical abused children's adjustment. Child Abuse \& Neglect 2000; 24: 641-651

24 Aken MAG van, Asendorpf JB, Wilpers S. Das soziale Unterstützungsnetzwerk von Kindern: Strukturelle Merkmale, Grad der Unterstützung, Konflikte und Beziehung zum Selbstwertgefühl. Psychologie in Erziehung und Unterricht 1996; 43: 114-126

25 Antonovsky A. Unraveling the mystery of health. How people manage stress and stay well. San Francisco: Jossey-Bass; 1987

26 Hurrelmann K. Gesundheitssoziologie. 6. Aufl. Weinheim: Juventa; 2006

27 Bettge S, Ravens-Sieberer U. Schutzfaktoren für die psychische Gesundheit von Kindern und Jugendlichen - empirische Ergebnisse zur Validierung eines Konzepts. Gesundheitswesen 2003; 65: 167-172

28 Ravens-Sieberer $U$, Wille $N$, Bettge $S$ et al. Psychische Gesundheit von Kindern und Jugendlichen in Deutschland. Ergebnisse aus der BELLAStudie im Kinder- und Jugendgesundheitssurvey (KiGGS). Bundesgesundheitsbl Gesundheitsforsch Gesundheitsschutz 2007; 50: 871878

29 Currie CE, Roberts C, Morgan A et al Young People's Health in Context. Copenhagen, Denmark: WHO Regional Office for Europe; 2004

30 Kurth B-M. Der Kinder- und Jugendgesundheitssurvey (KiGGS): Ein Überblick über Planung, Durchführung und Ergebnisse unter Berücksichtigung von Aspekten eines Qualitätsmanagements. Bundesgesundheitsbl Gesundheitsforsch Gesundheitsschutz 2007; 50: 533546

31 Richter M. Soziale Determinanten der Gesundheit im Spannungsfeld zwischen Ungleichheit und jugendlichen Lebenswelten: Der WHOJugendgesundheitssurvey. In: Richter M, Hurrelmann K, Klocke A, Melzer W, Ravens-Sieberer U, Hrsg. Gesundheit, Ungleichheit und jugendliche Lebenswelten: Ergebnisse der zweiten internationalen Vergleichsstudie im Auftrag der Weltgesundheitsorganisation WHO. Weinheim: Juventa; 2008; 9-37 
32 Kamtsiuris $P$, Lange $M$, Schaffrath Rosario A. Der Kinder- und Jugendgesundheitssurvey (KiGGS): Stichprobendesign, Response und Nonresponse- Analyse. Bundesgesundheitsbl Gesundheitsforsch Gesundheitsschutz 2007; 50: 547-556

33 Hölling H, Kamtsiuris P, Lange $M$ et al. Der Kinder- und Jugendgesundheitssurvey (KiGGS): Studienmanagement und Durchführung der Feldarbeit. Bundesgesundheitsbl Gesundheitsforsch Gesundheitsschutz 2007; 50: 557-566

34 Dölle R, Schaffrath Rosario A, Stolzenberg H. Der Kinder- und Jugendgesundheitssurvey (KiGGS): Datenmanagement. Bundesgesundheitsbl Gesundheitsforsch Gesundheitsschutz 2007; 50: 567-572

35 Ravens-Sieberer U. Der Kindl-R Fragebogen zur Erfassung der gesundheitsbezogenen Lebensqualität bei Kindern und Jugendlichen - Revidierte Form. In: Schumacher J, Klaiberg A, Brähler E, Hrsg. Diagnostische Verfahren zu Lebensqualität und Wohlbefinden. Göttingen: Hogrefe; 2003; 184-188

36 Erhart M, Hölling H, Bettge S et al. Der Kinder- und Jugendgesundheitssurvey (KiGGS): Risiken und Ressourcen für die psychische Entwicklung von Kindern und Jugendlichen - The German Health Interview and Examination Survey for Children and Adolescents (KiGGS): Risks and resources for the mental development of children and adolescents. Bundesgesundheitsblatt - Gesundheitsforschung - Gesundheitsschutz 2007; 50 (5-6): 800-809

37 Schwarzer R, Jerusalem M. Skalen zur Erfassung von Lehrer- und Schülermerkmalen. Dokumentation der psychometrischen Verfahren im Rahmen der Wissenschaftlichen Begleitung des Modellversuchs Selbstwirksame Schulen. Berlin: Institut für Psychologie, Freie Universität Berlin; 1999

38 Grob A, Lüthi R, Kaiser FG et al. Berner Fragebogen zum Wohlbefinden Jugendlicher (BFW). Diagnostica 1991; 37: 66-75

39 Donald CA, Ware JE. The measurement of social support. Research in Community and Mental Health 1984; 4: 325-370

40 Schneewind K, Beckmann M, Hecht-Jackl A. Familienklima-Skalen. Bericht. München: Institut für Psychologie - Persönlichkeitspsychologie und Psychodiagnostik der Ludwig Maximilians Universität München; 1985
41 Ravens-Sieberer U, Gosch A, Rajmil L et al, the European KIDSCREEN Group. The KIDSCREEN-52 Quality of Life measure for children and adolescents: Psychometric results from a cross-cultural survey in 13 European Countries. Value in Health 2007, doi:10.1111/j.15244733.2007.00291.x.

42 Goodmann R. The Strengths and Difficulties Questionnaire: A research note. Journal of Child Psychology and Psychiatry 1997; 38: 581-586

43 Nunnally JC, Bernstein IR. Psychometric Theory. 3. Aufl. New York, NY: McCraw-Hill; 1994

44 Cohen J. Statistical Power Analysis for the Behavioural Sciences. New York: Erlbaum; 1988

45 Anderman EM. School effects on psychological outcomes during adolescence. Journal of Educational Psychology 2002; 94 (4): 795-809

46 Torsheim T, Aaroe LE, Wold B. School-related stress, social support, and distress: prospective analysis of reciprocal and multilevel relationships. Scandinavian Journal of Psychology 2003; 44 (2): 153-159

47 Gazelle $H$. Class climate moderates peer relations and emotional adjustment in children with an early history of anxious solitude: A child x environment model. Developmental Psychology 2006; 42 (6): 11791192

48 Masten AS, Reed M-GJ. Resilience in development. In Snyder CR \& Lopez SJ, Eds. The handbook of positive psychology. Oxford: University Press; 2002; 74-88

49 Berkmann LF. The role of social relations in health promotion. Psychosomatic Medicine 1995; 57: 245-254

50 Wendt WR. Case Management im Sozial- und Gesundheitswesen. Eine Einführung. 2. Aufl. Freiburg i. B.: Lambertus-Verlag; 1999

51 Nestmann F. Soziale Netzwerke - soziale Unterstützung. In: Otto HU. Thiersch H, Hg. Handbuch Sozialarbeit/Sozialpädagogik. 2. Aufl. Neuwied: Lucherhand Verlag; 2001; 1684-1692

52 Labonte $R$. Community empowerment and leisure. Journal of Leisurability $1995 ; 23: 4-20$ 